\title{
RICERCHE SPETTROSCOPICHE SULLA SCINTILLA IN ATMOSFERA DI IDROGENO.
}

\author{
DotT. G. POLVANI.
}

\section{I. - Come varia nel tempo lo spettro della seintilla in atmosfera di idrogeno.}

$\S$ 1. - La disposizione sperimentale è essenzialmente quella stessa che usai per le ricerche sperimentali sulle variazioni nel tempo dello spettro della scintilla nell'aria; la descrizione è data nel Nuovo Cimento (Serie VI, Vol. XX, pagg. 119-184, nov.-dic. 1920). Qui mi limito ad esporre solo le modificazioni che fu necessario apportare per le nuove esperienze.

Lo spinterometro della scintilla in esame era contenuto in un opportuno recipiente ripieno di idrogeno. Questo, prodotto dalla reazione dell'acido solforico sullo zinco, veniva purificato con ripetuti lavaggi, prima attraverso una soluzione acquosa di permanganato e idrato potassico, poi di nitrato d'argento; ed infiue veniva essiccato facendolo gorgogliare abbondantemente e lentamente attraverso a dell'acido solforico concentrato. La pressione dell'idrogeno nel recipiente era di qualche centimetro d'acqua superiore alla pressione ambiente, per essere del tutto sicuro che nel recipiente non penetrasse l'aria ambiente per qualche eventuale imperfetta tenuta.

Le punte dello spinterometro in idrogeno erano d'alluminio.

Per ottenere che l'immagine della scintilla proiettata dallo specchio rotante lungo la fenditura dello spettrografo fosse limitata in ogni istante sempre alla stessa figura, non sono ricorso, come nelle passate esperienze, ad un diaframma posto vicinissimo alla scintilla in esame, ma invece ho prodotto con una buona lente acromatica un' immagine reale della scintilla sopra wa fenditura $g$ posta parallelamente all'asse della scin- 
tilla; e lo specchio rotante proiettava sulla fenditura dello spettrografo l'immagine della immagine della scintilla data dalla lente acromatica e opportunamente limitata dalla fenditura $g$.

La distanza della lente acromatica dalla scintilla in esame e quella dello specchio dalla fenditura limitatrice, erano ciaseuna il doppio della distanza focale rispettivamente della lente e dello specchio.

§ 2. - Al passaggio della scarica (oscillante o continua che fosse) l'idrogeno emetteva lo spettro della serie di Balmer; del secondo spettro nessuna traccia, ciò a causa del valore piuttosto grande della capacità.

Per la qualità delle lastre adoprate e per la durata data alle pose, negli spettrogrammi comparivano:

le $\mathrm{H}_{\beta}, \mathrm{H}_{r}, \mathrm{H}_{\delta}$ dell' idrogeno,

e le righe (in unità Angström) $\lambda=3587,3602,3612$, $3702,3713,3944,3962,4480,4513,4529,4663$ emesse dall'alluminio degli elettrodi.

Nella cernita degli spettrogrammi, per quanto riguarda la loro attendibilità circa lo studio delle variazioni subite nel tempo dallo spettro, mi sono attenuto allo stesso criterio esposto nel mio lavoro già citato (pag. 144).

\section{A. - Scariche oscillanti.}

$\S$ 3. - Periodo di un'oscillazione completa : $35 \times 10^{-6}$ sec.; quindi durata di una pulsazione luminosa: $17,5 \times 10^{-6}$ sec. $\left.{ }^{1}\right)$.

Durata completa della scintilla (come fenomeno luminoso): $2 \times 10^{-4}$ sec. circa $\left.^{2}\right)$.

1) Per il valore della capacità e del coefficiente di antoinduzione del circuito confronta il mio lavoro già citato (pag. 142).

2) Interponendo convenientemente uno specchio piano, verticale sui raggi di ritorno tra lo specchio concavo e la fenditura dello spettrografo, era possibile far cadere, sopra una lastra fotografica posta entro un'opportuna camera oscura l'immagine della scintilla data dallo specchio rotante. Nelle fotografie così ottenute comparivano una diecina di pulsazioni luminose, da cui si deduce che la durata della scintilla, come fenomenoluminoso, era appunto dell'ordine di grandezza sopra indicato. 
Distanza esplosiva: 2-3 $\mathrm{mm}$.

Numero delle scintille necessarie per impressionare la lastra: $1200-1500$.

Come nelle passate esperienze mi sono limitato allo studio delle variazioni che lo spettro della parte mediana della scintilla subisce durante le prime oscillazioni della scarica (2-3 a seconda della lastra).

§ 4. - Le righe dell'alluminio sopra ricordate posseggono, salvo una minore intensità, gli stessi caratteri già descritti nel caso delle scintille nell'aria; presentano tutte, eccetto la 3944, 3962 , spiccate variazioni periodiche d'intensità e intensità media decrescente; solo le 3944, 3962 subiseono invece variazioni periodiche appena sensibili e crescono leggermente in intensità media.

$\S$ 5. - La $\mathrm{H}_{\beta}$ non è molto intensa in confronto alle righe dell'alluminio; presenta variazioni periodiche $d$ 'intensità sensibilmente in fase con quelle dell'alluminio. Nei momenti di minimo non pare che raggiunga l'estinzione '). La sua inten. sità media passando dall' una alla successiva p!ılsazione Iuminosa rimane sensibilmente la stessa ${ }^{2}$ ).

1) Con questo non voglio escludere che in realtà non avvenga uno spegnimento in un tempo estremamente breve : però ciò sembra poco probabile (confronta $\oint 18$, pag. 21 ).

$\left.{ }^{2}\right)$ Ponendo al posto della lastra fotografica dello spettrografo, il vetro smerigliato, e spostando lo spinterometro sensibile in modo che sulla fenditura dello spettrografo cadessero le nltime pulsazioni luminose anzichè le prime è possibile accertarsi che $\mathrm{la}_{\beta}$ è ancora eccitata alla fine della scintilla.

L'esame ad occhio dello spettro fatto sul vetro smerigliato, o, meglio, con un oculare di grande apertura, a, per quello che riguarda l'aspetto delle righe, certamente assai ingannevole per la breve durata del fenomeno; pure da numerose osservazioni, credo di potere asserire che $\mathrm{H}_{\beta}$ mantiene il carattere pulsante fino alla fine della scintilla. (Anche $\mathrm{H}_{\boldsymbol{x}}$ presenta variazioni periodiche $d$ 'intensita; essa d molto meno espansa di $\left.H_{\beta}\right)$. 
La riga è molto espausa e appare come vuota nel suo interne per un largo solco d'intensità luminosa notevolmente minore, il quale la divide in due parti simmetriche '). Il solco i cui bordi non sono netti ma sfumati ed indecisi, è già formato, cioè non chiuso ${ }^{2}$ ) fîn dal principio della scintilla; è largo approssimativamente $15 \AA$. e si mantiene quasi ngualmente largo per le prime pulsazioni ${ }^{3}$ ).

In ciascuna di queste, la larghezza totale della riga, considerata in momenti equidistanti dal momento di massima larghezza, è sensibilmente la stessa e la larghezza totale massima ragginnta in ciascuna pulsazione è di circa $40 \AA$.

La $\mathrm{H}_{r}$ è meno intensa dell' $\mathrm{H}_{3}$ e presenta anch'essa variazioni periodiche d'intensitì ben marcate in fase con quella di $\mathrm{H}_{\beta}{ }^{3}$ ); l'intensità media passando dall' una alla successiva pulsazione luminosa rimane sensibrlmente la stessa.

La riga è molto espansa, molto più dell' $\mathrm{H}_{\beta}$; non appare vuota, ma anzi nel suo interno presenta un filetto leggermente più intenso che la divide simmetricamente. In ogni pulsazione luminosa la larghezza della riga, considerata in momenti equi-

1) Esaminando ad occhio la riga non mi à stato possibile osservare questa zona meno luminosa, nemmeno usando degli schermi assorbenti per attutire la troppo viva intensita luminosa. It da escludere però che il solco sia dovuto ad un effetto di sovraesposizione fotografica perchè esso si presenta anche in immagini estremamente deboli.

2) Nello studio delle variazioni nel tempo subite dalla seintilla tra elettrodi di calcio nell'aria, trovai (op. eit., pag. 149) che le righe 3934 3968 ( $\mathrm{K}, \mathrm{H}$ solare) erano antoinvertite; la prima fin dall'inizio della scintilla, la seconda un momento dopo. I solchi oscuri (chiari in negativa) delle inversioni delle $\mathrm{H}$ e $\mathrm{K}$ del calcio hamno aspetto assai diverso dal solco dell' $\mathrm{H}_{\beta}$; nel caso del calcio i solchi sono visibili anche ad occhio ed hanno bordi nettissimi; essi sono veramente zone pressochè buie. Nel caso dell' $\mathrm{H}_{\beta}$ si tratta invece solo di una zona di minore intensità luminosa.

$\left.{ }^{3}\right)$ Questo e i successivi dati relativi a larghezze di righe (o a parte di righe) si riferiscono ad una larghezza di mm. 0,08 della fenditura dello spettrografo.

4) Vale per la permanenza delle pulsazioni della $H_{\gamma}$ verso la fine della scintilla quanto ho gia detto per $1^{\prime} \mathrm{H}_{\beta}$ (vedi nota ${ }^{2}$ ) a pag. 5). L'esame ad occhio nel caso di $H_{Y}$ è però più difficile. 
distanti dal momento di massima larghezza è sensibilmente la stessa.

La larghezza totale massima raggiunta in ciascuna pulsasazione, è di circa $140 \AA$. e la larghezza del filetto di circa $10 \stackrel{\AA}{\text {. }}$

Al principio della scintilla la $\mathrm{H}_{r}$ è perciò appuntita, mentre la $\mathrm{H}_{\beta}$ ̀̀ bifida.

La $\mathrm{H}_{\delta}$ è estremamente espansa e debole assai più dell' $\mathrm{H}_{r}$. Presenta variazioni periodiche d'intensità in fase con quelle di $H_{\beta}, H_{\Upsilon}$; e non sembra anch'essa raggiungere nei minimi l'estinzione. La sua intensità media si mantiene inalterata per le prime pulsazioni luminose.

Dalla zona dell' $H_{\delta}$ fin verso i $3550 \AA$ A., si distende uno spettro continuo, sempre più debole andanclo verso le lunghezze d'onda più piccole, che presenta una larga zona di maggiore intensità in corrispondenza di $\mathrm{H}_{\varepsilon}$ ').

Sul fondo di questo spettro continuo appaiono le righe dell'alluminio dalla 3968 in poi. Esso presenta variazioni periodiche d'intensità ben marcate in fase con quelle di $\mathrm{H}_{\beta}$, $\mathrm{H}_{r}$ ecc. $\mathrm{L}$ 'intensità media si mantiene inalterata per le prime pulsazioni.

§ 6. - Se l'idrogeno è inquinato di una piccola quantità d'aria, appaiono negli spettrogrammi in corrispondenza della prima pulsazione luminosa le seguenti righe : 471s, 4703, 4701, $4652,4642,4631,4623,4609,4607,4601,4591,4470,4418$, $4385,4368,4317,4279,4266^{*}, 4253,4242,4236,4228,4176$, $4169,4155^{*}, 4119,4105,4085,4076,4072,4070,4067,4041$, $3995,3974,3956,3919,3912,3899^{*}, 3882,3830,3749^{*}, 3662^{*}$ che sono naturalmente righe d'aria ${ }^{2}$ ).

1) Sul fondo di questo spettro continuo appaiono le righe dell'allnminio dalle 3962 alle 3587 (vedi 9 ).

$\left.{ }^{2}\right)$ Quelle contrassegnate coll'asterisco sono di incerta determinazione perchè nebulose ed espanse; le altre sono date a meno di $\pm 2 \AA$. 
Esse presentano marcatissime variazionı periodiche d'intensità in fase con quelle delle righe dell'idrogeno e dell'alluminio e raggiungono nei minimi l'estinzione.

Esse sono tutte deboli, ed accetto le $4266^{*}, 4155^{*}, 3899^{*}$, $3749^{*}, 3662^{*}$ che sono nebulose e un poco espanse, sono tutte finissime.

La loro intensità media decresce rapidissimamente passando dall' una alla successiva pulsazione luminosa; tanto che alla seconda permangono solo le righe: 4718, 4703, 4701, 4418, $4266^{*}, 4155,4076^{*}, 4072,4070,3995,3899^{*}, 3749^{*}, 3662^{*}$ e alla terza e alla quarta solo le $4266^{*}, 4155^{*}, 3899^{*}, 3749^{*}$, $3662^{*}$ cioè le nebulose.

\section{B. - Scarica continua brusca.}

7. - Ricordiamo che questa è ottenuta ") sostituendo nel circuito di scarica del condensatore, all'elica di filo grosso di rame uua resistenza a liquido; le commnicazioni tra le varie parti del circuito sono fatte con larghi nastri metallici per ridurre quanto più è possibile l'autoinduzione del circuito.

La distanza esplosiva dello spinterometro nell' idrogeno è di circa $3 \mathrm{~mm}$. Il numero delle scintille necessarie ad impressionare la lastra fotografica è di circa un migliaio.

§ 8. - Le righe dell'alluminio presentano gli stessi caratteri già riscontrati nelle scintille brusche nell'aria '), anche rispetto alla durata.

$\mathrm{L}^{\prime} \mathrm{H}_{\beta}$ non appariva molto intensa; la sua durata era di circa $20 \times 10^{-8}$ sec.; cioè dell'ordine di grandezza della durata delle due righe 3944,3962 dell'alluminio $\left.{ }^{3}\right)$. Essa è espansa e nebulosa; e presenta nel suo interno il solco già descritto nel caso delle scariche oscillanti. Questo solco presenta il suo

1) Vedi G. Polvani. - op. eit., pag. 172.

') Idem. - Op. cit,. pag. 177.

3) Idem. - Op. cit., pag. 177. 
massimo di larghezza (circa $15 \AA$.) proprio all'inizio della scintilla e va un poco lentamente stringendosi nel tempo apparendo così come un $\mathrm{V}$ d'angolo strettissimo (il vertice del $V$, ciò̀ la chiusura del solco, non appare in fotografia); la larghezza totale e massima della riga (circa 40 A.) e il massimo d'intensita luminosa di essa sono sensibilmente simultanee e poco dopo l'inizio della scarica (due milionesimi di secondo circa).

$\mathbf{L}^{\prime} \mathbf{H}_{\mathbf{r}}$ è meno intensa della $\boldsymbol{H}_{\beta}$; la sua durata è pressochè uguale a quella dell' $H_{\beta}$. Essa presenta nel mezzo, come nel caso delle scariche oscillanti, il filetto più intenso. È molto più espansa dell' $\mathrm{H}_{\beta}$; il momento di massima larghezza (circa 140 A.) e quello di massima intensità sono contemporanei tra loro e ai corrispondenti dell' $H_{\beta}$. All' inizio della scarica l' $\mathrm{H}_{\gamma}$ è appuntita, mentre $\mathrm{l}^{\prime} \mathrm{H}_{3}$ è bifida.

$\mathrm{L}^{\prime} \mathrm{H}_{\delta}$ è estremamente espansa e molto debole.

La luminosità delle varie righe si manifesta contemporamente ed in modo brusce.

Si osserva poi e solo all'inizio della scarica uno spettro continuo sempre più debole verso il violetto estremo, che si estende dalla zona dell' $\mathrm{H}_{\delta}$ fin verso i $3550 \AA$.

Se l'idrogeno è inquinato di piccole quantità d'aria, appaiono numerose righe d'aria di durata molto viù breve ed intensità assai più debole in confronto di quelle dell'alluminio e dell' idrogeno.

§9. - Il confronto di questi risultati sperimentali, con quelli già ottenuti nelle precedenti mie esperienze nel caso di scintille nell'aria ambiente, mostra che $i$ caratteri principali che differenziano il comportamento delle righe dell'idrogeno da quello delle righe d'aria sono:

a) il grande allargamento delle righe deIl' idrogeno di contro alla generale finezza delle righe d'aria (beninteso queste eccitate nelle medesime condizioni elettriche, e con la scintilla nell'aria); 
b) la presenza anche nelle ultime pulsazioni luminose della scintilla, delle righe dell'idrogeno, di contro alla mancanza di quelle d'aria (queste eccitate ecc.), le quali sono in generale assai già indebolite o addirittura estinte passando alla seconda e alla terza pulsazione luminosa.

Questi due caratteri che differenziano il comportamento delle righe dell' idrogeno da quello delle righe d'aria, mi hanno suggerito le esperienze che espongo nei paragrafi che seguono.

\section{II. - L' allargamento delle righe dell' idrogeno e r'efretto stark-Lo Surdo.}

$\$ 10$. - Secondo le vedute moderne, l'allargamento che si ottiene nelle righe del primo spettro dell' idrogeno, eccitato con le scariche di un condensatore, viene, con buone argomentazioni, riconnesso alla scissione subita dalle stesse righe spettroscopiche per l'effetto Stark-Lo Surdo, e si attribuisce appunto all'azione che sull'atomo lumiuoso ha il campo elettrico prodotto dalle particelle elettrizzate che gli sono vicine.

Stark che propose per primo questa spiegazione dell'allargamento delle righe, si appoggia soprattutto sul fatto che la risoluzione delle righe di una serie cresce col crescere del numero del termine e che l'allargamento cresce in simile maniera seguendo anche le eventuali dissimetrie della risoluzione elettrica. E certamente gli studii del Merton, del Merton e Nicholson ') sulla distribuzione della intensità luminosa nella larghezza stessa della riga allargata, eseguiti su alcune righe dell' idrogeno, dell'elio ece. avvalorano molto l'ipotesi che l'allargamento, il quale si presenta notevole nel caso delle scariche di un condensatore, sia dovuto ad un effetto di campo elettrico.

1) T. R. Merton: Prooeed. of the Roy. Soc. of London (1916), p. 322. T. R. Merton o J. W. Nicholson. Philos. Trans. of the Roy. Soc. of London. Ser. A., vol. 216, pag. 459-488, (1916). 
Secondo questa interpetrazione, il filetto di maggior, 'ntensità luminosa ed il soleo.di minore intensita, i qua' rispettivamente appaiono nell' $\mathrm{H}_{\Upsilon}, \mathrm{H}_{\beta}$, sono da attribuirsi q!. lo alla presenza, questo all'assenza di componenti centrali is to intense nella scissione, nell'effetto Stark-Lo Surdo, rispet : : amente di $\mathbf{H}_{\boldsymbol{r}}$ e di $\mathbf{H}_{3}$.

In teoria, nel caso della scintilla, al campo molecola:.. o meglio all' insieme di tutti i campi molecolari che si es jitano confusamente in tutte le direzioni, si può pensaru. he si sovrapponga almeno al primo inizio della starica il $*$. jo prodotto dalla differenza di potenziale applicata agli est . ni dello spinterometro. Ho detto solo «al primo inizio 》pu hè immediatamente dopo, quando lo spazio esplosivo è già : co di joni, la differenza di potenziale degli elettrodi si riı.. rà subito incomparibilmente più piccola di quella iniziale. iò rispetto alle variazioni nel tempo; quanto alle variazioni aziali, è naturale pensare che la maggiore intensità di c: ' yo si debba trovare in vicinanza degli elettrodi, specialn : te subico dopo l'inizio della scarica.

Con questi concetti ho ricereato, modificando corrispor $n$ temente in due modi la disposizione sperimentale, se ef . tivamente si presentava, nella scintilla in idrogeno, il fenol no Stark-Lo Surdo per effetto del campo impresso.

\section{A. - Prima modifieazione.}

$\S 11 .-$ Immediatament $\leftrightarrow$ accanto alla fenditura limitatr $\Rightarrow g$ (vedi parag. 2), tra questa e lo specchio rotante interpos: ni raggi luminosi provenienti dalla scintilla, un grosso spai.. di Islanda disposto in modo che fosse orizzontale il piano . . ב I'asse cristallografico principale e della direzione di propicyazione dei raggi; e all' unica fenditura dello spettrografi ;ostitnii due fenditure verticali, parallele, ugualmente sottili e la cui distanza era uguale alla distanza di due punti corrispondenti delle dne immagini della scintilla proiettate dallo 
specchio concavo sul collimatore. Verificavo se quest' ultima condizione (quella della distanza delle fenditure) era soddisfatta guardando attraverso allo spato l'immagine, data a fermo dallo specchio, delle due fenditure (opportunamente illuminate con luce riflessa sulla prima faccia del primo prisma dello spettrografo) ed osservando se essa appariva come formata di tre fenditure (non di quattro) di cui quella interna più luminosa ').

Le due immagini alla Feddersen della scintilla venivano dallo specchio rotante proiettate esattamente lungo le due fenditure, e sulla lastra fotografica si ottenevano così le immagini di due spettri $S_{p}, S_{s}\left(S_{p}\right.$ indica lo spettro ottenuto con luce polarizzata, all'emergenza dallo spato, parallelamente al campo elettrico dello spinterometro e $\mathbf{S}_{s}$ l'altro spettro), spostati l' uno rispetto all'altro ed in parte sovrapposti. Però la separazione operata dallo spato si adattava bene e con la distanza esplosiva dello spinterometro di solito usata (3-4 mm.) e con la dispersione posseduta dallo spettrografo: infatti le due immagini proiettate dallo specchio sulle fenditure erano nettamente separate l'una dall'altra e le due righe $\mathrm{H}_{\beta}$ e la $H_{\gamma}$ dello spettro $\mathrm{S}_{p}$ non si sovrapponevano ad altre righe e solo la rimanente $H_{r}$ si sovrapponeva, con uno dei suoi bordi, alla riga (fine) 4480 dell'alluminio appartenente a $\mathbf{S}_{p}$.

Per togliere poi l'inconveniente della diversa intensità Iuminosa che avrebbero avuto i due spettri a causa del diverso angolo tra piani di polarizzazione dei due fasci polarizzati e

') É pure anche necessario che lo specchio riceva tanta luce dell' uno quanto dell'altro dei due fasci emergenti dallo spato. Ciò si ottiene spostando accortamente in senso orizzontale lo spinterometro della scintilla in esame (e conseguentemente la lente acromatica e la fenditura limitatrice); e si può verifieare se la condizione è soddisfatta lasciando passare attraverso ad un nicol (di grande apertura) prima solo uno, poi solo l'altro fascio polarizzato ed osservando se, allo scoccare della scintilla la macchia luminosa, vista sopra un vetro smerigliato posto verticalmente dietro allo specchio (fermo) assume, rispetto allo specchio e alla verticale due posizioni simmetriche. 
piano d'incidenza sullo specchio e sui prismi, interposi tra spato e specchio una lastrina di quarzo a facce parallele così grossa da ottenere una rotazione dei piani di polarizzazione di circa $45^{\circ}$ per la zona dello spettro compreso tra $H_{\beta}$ e $H_{\gamma}$

Infine la lente collettrice posta sulla testata del collimatore e che originariamente era di quarzo, fu sostituita con una di vetro di uguale potere diottrico ').

Ora se si pensa alla maniera nella quale nell'effetto StarkLo Surdo trasversale si scompongono le righe dell'idrogeno appartenenti alla serie di Balmer, e alla maniera nella quale sono polarizzate le varie componenti, si intende subito che se nella scintilla vi fosse un eventuale effetto Stark-Lo Surdo, prodotto dal campo elettrico applicato alle punte dello spinterometro, le righe dell'idrogeno dovevano apparire nei due spettri $\mathrm{S}_{p}, \mathrm{~S}_{\mathrm{s}}$ con diverso aspetto; e precisamente le $\mathrm{H}_{\beta}$ e $\mathrm{H}_{r}$ di $\mathrm{S}_{i}$ dovevano essere più espanse della $\mathrm{H}_{\beta}$ e della $\mathrm{H}_{r}$ di $S_{p}$ ed in particolare questa diversità di larghezza dovera mostrarsi nei solchi delle due $\mathrm{H}_{\beta}$.

Invece, sia nella scarica oscillante come in quella continua brusca, le $\mathrm{H}_{3}$ dei due spettri $\mathrm{S}_{p}, \mathrm{~S}_{s}$ non mostrano, nemmeno all' inizio della scintilla, sensibile diversità di aspetto nè tra loro nè con le $\mathrm{H}_{\beta}$ degli spettrogrammi ottenuti con una sola fenditura e senza spato. Lo stesso dicasi per $\mathrm{H}_{r}$.

Questa prima modificazione della disposizione sperimentale serve bene per seguire da istante a istante l'andamento nel

1) Il numero delle scintille necessarie ad impressionare la lastra era di eirca 2500. E veramente notevole come per un si gran numero di scintille la concordanza delle immagine proiettate sulla fenditura si mantenga ottima.

L'osservazione delle immagini fatte per luce diffusa sulla testata del collimatore, diventò, in questa disposizione, incerta per insufficienza d' intensità; fu necessario per decidere della concordanza o meno delle stesse immagini puntare un viseur sull' immagine della fenditura data per riflessione dalla prima faccia del primo prisma dello spettrografo e riferire l'inizio delle immagini al reticolo del viseur. 
tempo di tutto il fenomeno, ma non permette l'esame della scintilla su tutto l' intervallo esplosivo ed in particolare presso gli elettrodi. La seconda modificazione ha invece di mira lo studio ad un certo istante di tutto l'intervallo esplosivo.

\section{B. - Seconda modificazione.}

\$12. - I due spinterometri, quello della scintilla in esame e quello sensibile, furono entrambi girati da orizzontali a vercali; e il primo fu situato di fianco e all'altezza del collimatore. Lo specchio rotante fu posto in modo che il suo asse di rotazione fosse verticale rimanendo però il suo centro di figura nella stessa posizione di prima rispetto al collimatore. Anche la fenditura della lanterna fin girata verticalmente.

Come nella prima modificazione, tra la scintilla in idrogeno e lo specchio furono interposte nella stessa posizione e con lo stesso scopo di prima, la lente acromatica, la fenditura limitatrice $g$ (girata verticalmente), lo spato d'Islanda girato anch'esso di $90^{\circ}$ gradi intorno alla direzione di propagazione dei raggi e la lamiua di quarzo. Al collimatore fu lasciata la sua unica fenditura.

Il fuuzionamento poi di tutto l'apparechio sperimentale el'a il solito: la scurica veniva provocata a tempo con I'illuminazione, per mezzo dello specchio rotante, dello spinteroinatro sensibile, e lo specchio, vedendo attraverso lo spato l'im. magine della scintilla data dalla lente acromatica, proiettava sulla testata del collimatore, trasversalmente alla fenditura, una sotto l'altra, dne immagini alla Feddersen della scintilla.

Lo spinterommtro sensibile era messo in punto tale che le immagini si inizistssero proprio sulla fenditura dcl collimatore - la separazione dello spato era cosi forte che le tue immagini risultavano nettamente distaccate. Cosi lo spettroscopio fornira i lue spettri $S_{p}, S_{s}$ uno sotto l'altro, e la scintilla cra quindi esaminata nel sno inizio (cioè nell' istante più 
favorevole) su tutta la lunghezza dell'intervallo esplosivo. Questa seconda disposizione completa dunque la prima.

Le fotografie mostrano che $\mathrm{i}$ due spettri $\mathbf{S}_{p}, \mathrm{~S}_{s}$ non presentano sensibili differenze tra loro; in particolare nel medesimo punto dell'intervallo esplosivo i solchi delle due righe $\mathbf{H}_{\beta}$ sono ugualmente larghi e i filetti delle due $H_{\gamma}$ sono ugualmente intensi e ciò tanto con scariche oscillanti quanto con scariche continue brusche.

\section{$\S 13 .-$ Dunque:}

- il campo elettrico proveniente dalla differenza di potenziale applicato allo spinterometro non dà origine ad un effetto Stark-Lo Surdo sensibile o almeno distinguibile dalle alterazioni dell'aspetto delle righe dell'idrogeno, che come abbiamo detto sopra, sono state ragionevolmente attribuite ad un effetto Stark-Lo Surdo dei campi molecolari,

- e se veramente l'allargamento, e nel caso di $\mathbf{H}_{\beta}$ il solco e nel caso di $\mathrm{H}_{\gamma}$ il filetto, si debbono secondo l'idea di Stark attribuire ai campi elettrici molecolari, bisogna am. mettere che questi allo scoccare della scintilla assurgano immediatamente al loro valor massimo e decrescano poi assai lentamente.

\section{III. - Sull' origine dell' emissione luminosa dell' idrogeno nella scintilla.}

\$ 14. - L'altro carattere oltre quello della grande espan. sione che differenzia le righe dell'idrogeno da quelle d'aria, eccitato in eguali condizioni elettriche con la scintilla nell'aria, è la loro presenza anche nelle ultime pulsazioni luminose della scintilla.

Occorre che ricordi brevemente i risultati ben noti ottenuti dalla indagine fatta con lo specchio rotante (e metodi 
simili) sulla scintilla oscillante nell'aria. Ad ogni mezza oscillazione della scarica, partono dagli elettrodi e prevalentemente del catodo, ciuffi luminosi di vapori metallici con velocità non molto grande (in media un migliaio di $\mathrm{m}$. al sec.) e decrescente. Questa emissione è più o meno ricca a seconda della capacità e dell'autoinduzione del circuito di scarica e della qualità degli elettrodi.

Iu pari tempo in ciascuna delle prime mezze oscillazioni della scarica (3-6 mezze oscillazioni, dipendentemente dagli elementi elettrici del circuito e dal metallo degli elettrodi e per una durata un poco inferiore al semiperiodo della scarica) appare, sempre con frequenze elevate, e può mancare con frequenze basse, una colonna luminosa che unisce istantaneamente l' uno all'altro elettrodo, certamente dovuta a luminescenza, provocata dal passaggio della scarica, del solo gas ambiente.

La scintilla poi in opportune condizioni, si inizia con un filetto sottilissimo - detto pilota - che attraversa istantaneamente tutto lo spazio esplosivo ed ha termine con le code, zone non molto luminose, le quali sogliono attribuirsi a vapori o gas rimasti incandescenti dopo la fine della scarica.

Naturalmente questi elementi della scintilla variano profondamente al variare degli elementi elettrici del circuito di scarica.

Ora, il fatto che nelle mie ricerche sulle variazioni spettroscopiche della scintilla nell'aria, le righe dovute a questa apparivano in generale deboli o addirittura estinte alla terza e quarta pulsazione luminosa ${ }^{1}$ ), si accorda bene con l'altra della esistenza solo alle prime mezze oscillazioni della scarica delle colonne luminose istantanee dovute a luminescenza gassosa. E il diverso comportamento del gas idrogeno suggeriva appunto di ripetere l'esperienze di Feddersen sn scintille in atmosfera di idrogeno.

1) Vedi op. cit., pag. 167. 
§ 15. - Questo è ciò che ho fatto, variando però opportunamente la parte ottica della disposizione di Feddersen, in modo da riuscire con lo studio delle varie immagini monocromatiche della scintilla dovute alle luci delle $\mathrm{H}_{x}, \mathrm{H}_{3}, \mathrm{H}_{\gamma}$ e a quella di qualche riga metallica, a mettere in evidenza l'origine della Iuminosità dell'idrogeno.

Perciò tra scintilla e specchio rotante interposi uno spettroscopio a visione diretta dell'Amici (composto per l'oceasione con lenti di quarzo di grande apertura e un buon prisma d'Amici ')) sfornito di fenditura ed oculare e che possedeva grande luminonità $\theta$ forte potere dispersivo. Nel piano focale della lente collimatrice si trovava parallelamente alle costole dei prismi, la scintilla in idrogeno; e nel piano focale della lente obbiettiva, sul quale si formavano le varie immagini monocromatiche della scintilla, era collocata una fenditura (anch'essa parallelamente alle costole dei prismi) di larghezza regolabile e che poteva essere spostata parallelamente a sè stessa in modo da lasciar passare solo la luce di una certa zona scelta dello spettro.

La forte dispersione dell'Amici e un'accorta scelta di metalli per gli elettrodi mi permise di ottenere che le immagini monocromatiche dovute alle $\mathrm{H}_{\alpha}, \mathrm{H}_{3}, \mathrm{H}_{\Upsilon}$, e a qualche riga metallica non si sovrapponessero tra loro, nè ad altre immagini monocromatiche e quindi mi permise di tener larga la fenditnra tanto da avere pura e completa l'immagine monocromatica scelta.

Lo specchio rotante (mosso da un motorino elettrico) era concavo e distava dalla feuditura il doppio della distanza focale, e aveva il centro di figura nel piano verticale passante per l'asse ottico dello spettroscopio e l'asse di rotazione parallelo alle costole dei prismi. Il motorino poteva essere con opportuno congegno alzato ed abbassato così da portare lo

1) Questo si poteva collocare e togliere dal suo posto; in modo da permettere anche l'osservazione dell' immagine di Feddersen non monocromatizzata. 
specchio all'altezza voluta perchè fosse investito per intero dal fascio monocromatico uscente dalla fenditura. Non fu possibile per insufficienza $d$ 'intensità luminosa ottenero le fotografie delle varie immagini monocromatiche ') le quali perciò vennero osservate ad occhio raccogliendole sopra un vetro smerigliato, o, meglio, per avere maggiore chiarezza, osservandole coll'aiuto di una grande lente di campo $(15 \mathrm{~cm}$. di diametro e 20 di distanza focale) collocata al posto del vetro smerigliato, ponendo allora l'occhio dove la stessa lente forniva l'immagine, assai impiccolita, dello specchio.

L' uso della lente del campo, è vantaggiosissimo perchè permette di avere impressioni vivissime e di osservare i più minuti particolari delle immagini i quali vanno persi o risultano offuscati con l' uso del vetro smerigliato.

Infine per ottenere che i raggi riflessi dallo specchio incidessero sulla lente del campo sono ricorso al comune artificio della bacchetta metallica solidale allo specchio e rotante fra due punti inseriti nel circuito di scarica.

§ 16. - Esporrè come esempio i resultati ottenuti con elettrodi di argento ${ }^{2}$ ). Le condizioni del circuito di scarica ${ }^{3}$ ) furono scelte in modo che nell'immagine, osservata senza prisma dell'Amici e senza fenditura limitatrice, fossero presenti insieme e ben distinti i vari elementi della scintilla: pilota, colonne, ciuffi, code *). La scintilla pilota e le colonne (queste

1) Non à possibile ottenere le fotografie delle immagini monocromatiche facendo cadere successivamente, col solito artificio dell'effetto fotoelettrico, molte immagini le une sulle altre perche la forma delle scintille specialmente se un po' lunghe à variabilisima.

2) Osservando con uno spettroscopio graduato la scintilla riconobbi che dell'argento apparivano solo lo righe 5212, 5465, 5865, e, dovute ad impurezza, le $\mathrm{D}_{1}, \mathrm{D}_{2}$; righe tutte assai lontane da quelle dell'idrogeno. L'argento si presta quindi assai bene a queste esperienze.

2) Capacità : $10^{-1}$ forad; coefficiente d'autoinduzione: $10^{-4}$ henry circa.

4) Queste ultime con elettrodi d'argento, sono invero assai misere; il magnesio ne da invece delle ricchissime, color verde (dovuto alla riga $b$ ). 
assai numerose) sono di color fra rosa e porpora; i ciuffi (più intensi presso gli elettrodi e aucora più intensi e ricchi quelli del catodo) sono bianchi, tendenti al giallo-verde, con colorazione via via più visibile; infine le code sono anch'esse giallo•verdastro chiaro.

L'esame delle immagini monocromatiche (con eguali condizioni di scarica) ba dato $\mathrm{i}$ seguenti risultati:

Immagine dovuta all' $\mathrm{H}_{x}$. - Si vedono la scintilla pilota, le colonne (queste in gran numero) e si distingono ancora, benchè un po' più corti, i ciuffi col loro caratteristico andamento curvo, più intensi presso gli elettrodi e ancor più intensi e ricchi quelli del catodo e tra loro via via sempre meno intensi. Le code sono assai esigue e rare.

Immagine dovuta all' $\mathrm{H}_{\beta}$ o all $\mathrm{H}_{\boldsymbol{\gamma}}$. - Prescindendo dalla diversa colorazione, l'aspetto di queste immagini è simile a quello dell'immagine data dalla $\mathrm{H}_{x}{ }^{1}$ ).

Immagine dovuta alla riga 5465 (verde dell'argento). - Si distinguono i ciuffi e le code, gli uni e le altre più ricchi ma con aspetto ed andamento simili a quelli osservati nelle imimagini dáte dalle $\mathrm{H}_{\alpha}, \mathrm{H}_{\beta}, \mathrm{H}_{\gamma}$; manca la pilota.

$L$ 'immagine dovuta alla riga 5865 (gialla dell'argento) $e$ alle $\mathrm{D}_{1}, \mathrm{D}_{2}$ (dovute ad impurezá) prese tutte e tre insieme ̀̀ simile a quella data dalla $\mathbf{5 4 6 5}$.

Usando magnesio ${ }^{8}$ ), alluminio ${ }^{3}$ ), platino, zinco nelle medesime condizioni di scarica o variando sia con questi metalli,

1) Con l' $\mathrm{H}_{Y}$ l'osservazione è un po' penosa, l'immagine non essendo netta (certo per la grande espansione della riga).

9) Ecco, come altro esempio, i risultati ottenuti con elettrodi di magnesio: le immagini dovute ad $\mathrm{H}_{\alpha}, \mathrm{H}_{\beta}$ presentano la pilota, le colonne (assai numerose), i ciuffi (al solito più intensi presso gli elettrodi e ancor più intensi e ricchi quelli del catodo, con aspetto simile a quello possednto dai ciuff, che sono però più Iunghi, dell' immagine data dalla $b$ ) e le code (queste assai corte mentre invece quelle date dalla $b$ sono lunghissime). La riga 4481 dà origine ad un'immagine costituita da soli ciuffi cortissimi, molto intensi e poco numernsi; tanto con la 4481 come con la $b$ non si scorge la pitota.

$\left.{ }^{3}\right)$ L'Alluminio si presta molto bene per l'osserrazione dell'immagine data da $\mathrm{H}_{\beta}$. 
sia anche coll'argento, le condizioni di scarica si ottengono risultati simili ai precedenti.

Le immagini dovute alle righe dell'idrogeno posseggono pilota, colonne, ciuffi e code; quelle dovute a righe metalliche mancano di pilota ${ }^{1}$ ) ed hanno ciuffi e code più o meno ricchi e lunghi (le code possono mancare addirittura, ad esempio nell' immagine data dalla 4481 del magnesio, o essere straordinariamente lunghe come nel caso della $b$ ).

Si nota poi sempre una stretta somiglianza di forma e di posizione ed uguali particolarità circa la distribuzione dell' in. tensitat $\left.{ }^{2}\right)$ tra i ciuffi e le code dovute a righe dell'idrogeno e i ciuffi e le code dovute (nelle medesime condizioni di scarica) a righe del metallo, ciò che mostra esservi lungo il percorso dei vapori metallici proiettati dagli elettrodi e contemporaneamente all'emissione luminosa di questi, anche emissione luminosa da parte dell' idrogeno.

In particolare nelle condizioni delle esperienze descritte nei paragrafi 1-13, le immagini dovute alle righe dell'idrogeno posseggono larghe colonne e ciuffi ricchissimi e intensi; colonne e ciuffi che si distingono male le une dagli altri causa la piccola distanza esplosiva e la moderata velocità dello specchio.

Perciò lo studio esposto in quei paragrafi si riferisce alla luminescenza globale dell'idrogeno senza distinguere quella

') Non dico anche di «colonne », perchè nel caso del $\mathrm{Mg}$ l'immagine data dalla $b$ pare che possegga delle debolissine colonne anche nella prima mezza oscillazione della searica. - Forse esse non sono dovute proprio alla $b$, ma alla porzione, vicina alla $b$, del debole spettro continuo che accompagna l'emissione di righe; o forse a riaccensione di particelle di $\mathrm{Mg}$ (o di ossido di $\mathrm{Mg}$ dovuto ad imporezza dell' $\mathrm{H}$ ) rimaste sospese nell'intervallo esplosivo dalle scariche precedenti. La cosa meriterebbe forse un esame più aceurato.

2) In opportune condizioni di scarica (capacità e autoinduzione molto piccole) l'emissione di vapori metallici, si può ridurre a pochissimo come si riconosce dalla poverti dei ciuffi si osserva allora che alle ultime oscillazioni la scintilla è costituita solo di colonne dovute a luminescenza del gas idrogeno. 
delle colonne da quella dei ciuffi e ciò spiega perchè in quelle esperienze manchino nei minimi veri momenti di estinzione nelle righe dell' idrogeno.

Nella scarica continua brusca, tanto le immagini monocromatiche dovute a righe metalliche, quanto quelle dovute a righe dell'idrogeno si riducono a brevissimi nastri luminosi che terminano con code più o meno lunghe a seconda della riga considerata; quelle dovute all'idrogeno sono di media lunghezza.

§ 17. - Tutto ciò porta alla conclusione che nella scintilla in idrogeno l'emissione dello spettro di questo gas ha due origini diverse: una non dissimile a quella delle righe d'aria nelle solite scintille ed una che va di pari passo colla luce dei vapori metallici.

La prima è rappresentata dalla scintilla pilota e dalle colonne; la seconda dai ciuffi e dalle code.

Il trovare questa seconda specie di luminosità dell'idrogeno sconcerta un po', perchè mentre è facile comprendere come i vapori metallici emessi dagli elettrodi diano tale apparenza, altrettanto difficile è spiegarla per il gas ambiente.

Perciò ho insistito nelle osservazioni, che mi hanno maggiormente convinto dello stretto legame lungo i ciuffi tra la emissione luminosa metallica e quella del gas idrogeno come risulta dalla contemporaneità delle due emissioni e dal fatto ehe dei ciuffi dovuti all'idrogeno come dei ciuffi dovuti al metallo, sono più intensi quelli che provengono dal catodo.

Come dunque si può interpretare tutto ciò?

La prima idea che si presenta è che l'idrogeno dei ciuffi sia occluso (o combinato) negli elettrodi e che da questi si liberi nel momento delle scariche parziali; ma se tale fatto sembra assai verosimile non è chiaro quale relazione abbia coi fenomeni osservati, perchè infine tutto lo spazio è già pieno d'idrogeno e non si tratta qui di spiegarme la presenza in 
certi elementi, ma sibbene il comportamento della sua luminosità.

Appunto per questo non ho potuto non cedere alla tentazione di prendere in considerazione la particolarità descritta, al lume delle idee recentemente proposte sulla struttura atomica e le righe spettroscopiche, quali per esempio il lettore può trovare assai chiaramente esposte nel libro «Atombau e Spektrallinien » del Sommerfeld.

Se ci si pone da tal punto di vista, l'atomo-jone di idrogeno non essendo che un nucleo positivo atomico e man cando dell'elettrone satellite, non potrebbe dare l'emissione di righe, la quale già da tempo per la maggior parte degli altri elementi si attribuisce appunto ad atomi-joni positivi; ma la darebbe quando dopo essere stato violentemente attratto. dal catodo se ne distaccasse di nuovo avendo da questo ricevuto il suo elettrone satellite, gravitante in salti successivi dall'una altra orbita secondo le idee di Bohr.

Tale concetto spiegherebbe dunque bene i ciuff idrogenici provenienti dal catodo che sono $\mathrm{i}$ più intensi. E quelli, sia pure meno intensi, provenienti dall'anodo?

Con animo grato al prof. Luigi Puccianti pongo anche a questo seritto la data:

Istituto di Fisica della R. Università

Pisa, maggio-gingno 1921. 